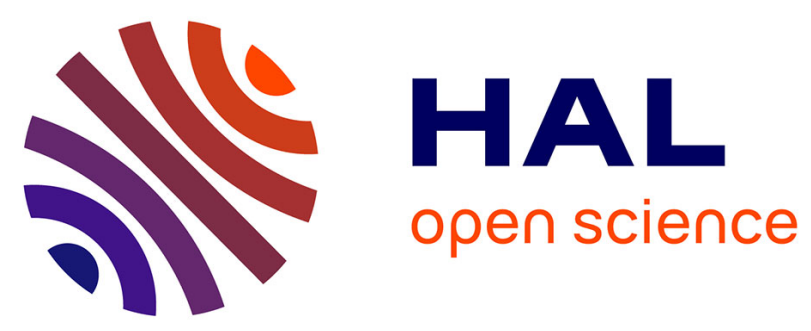

\title{
Performances biomécaniques primaires des ostéosynthèses arthroscopiques de glène
}

Philippe Clavert, Florence Aim, Nicolas Bonnevialle, Marine Arboucalot, Matthieu Ehlinger, Thomas Bauer

\section{- To cite this version:}

Philippe Clavert, Florence Aim, Nicolas Bonnevialle, Marine Arboucalot, Matthieu Ehlinger, et al.. Performances biomécaniques primaires des ostéosynthèses arthroscopiques de glène. Revue de Chirurgie Orthopédique et Traumatologique, 2019, 105, pp.157 - 160. 10.1016/j.rcot.2018.12.024 . hal-03486528

\section{HAL Id: hal-03486528 \\ https://hal.science/hal-03486528}

Submitted on 20 Dec 2021

HAL is a multi-disciplinary open access archive for the deposit and dissemination of scientific research documents, whether they are published or not. The documents may come from teaching and research institutions in France or abroad, or from public or private research centers.
L'archive ouverte pluridisciplinaire HAL, est destinée au dépôt et à la diffusion de documents scientifiques de niveau recherche, publiés ou non, émanant des établissements d'enseignement et de recherche français ou étrangers, des laboratoires publics ou privés.

\section{(ㄷ)(1) $\$$}

Distributed under a Creative Commons Attribution - NonCommercial| 4.0 International 


\section{Mémoire original}

\section{Performances biomécanique primaire des ostéosynthèses arthroscopiques de} glène.

\section{Biomechanical properties of transosseous bony Bankart repair in a cadaver model}

Philippe Clavert ${ }^{1,2,3}$, Florence Aim ${ }^{4}$, Nicolas Bonnevialle ${ }^{5}$, Marine Arboucalot ${ }^{5}$, Matthieu Ehlinger ${ }^{1,6}$, Thomas Bauer ${ }^{4}$ et la SOFCOT ${ }^{7}$

1. Laboratoire ICube - GEBOAS - UMR 7357, CNRS UMR 7357, Equipe 12 Matériaux Multi-échelles et Biomécanique, Institut de Mécanique des Fluides et des Solides, 2 - 4 rue Boussingault, 67000 Strasbourg, France

2. Institut d'Anatomie Normale, Faculté de Médecine, Fédération de Médecine Translationnelle, FMTS, 4 rue Kirschleger, 67085 Strasbourg Cedex, France

3. Service de chirurgie du membre supérieur, Hôpitaux Universitaires de Strasbourg, CCOM, Avenue Baumann 67400 Illkirch, France

4. Service de chirurgie orthopédique, CHU Ambroise Paré, AP-HP, 9 avenue Charles de Gaulle, 92100 Boulogne, France

5. Département de chirurgie orthopédique, hôpital Riquet, $\mathrm{CHU}$ de Toulouse, place Baylac, 31059 Toulouse cedex, France

6. Service de chirurgie orthopédique et de traumatologie, Hôpital Universitaire de Strasbourg, Hôpital de Hautepierre, 1 Avenue Molière, 67098 Strasbourg Cedex, France

7. 56 rue Boissonnade, 75014 Paris Cedex, France

Correspondance : $\quad$ Philippe CLAVERT

Institut d'Anatomie Normale,

Faculté de Médecine

4 rue Kirschleger

67085 Strasbourg Cedex, France.

+33388552151

philippe.clavert@chru-strasbourg.fr

Ne pas utiliser, pour citation, la référence française de cet article, mais celle de l'article original paru dans Orthopaedics \&Traumatology: Surgery \& Research, en utilisant le DOI ci-dessus. 


\section{Abstract}

2 Introduction : A partir d'un modèle biomécanique de luxation antérieure de l'épaule avec

3 une fracture du bord antéro-inférieur de la glène correspondant à une fracture Eideberg IA,

42 techniques d'ostéosynthèse arthroscopique ont été évaluées et comparées à un groupe

5 témoin.

6 L'hypothèse de cette étude était que l'ostéosuture permettait de stabiliser de façon

7 suffisante et efficace une fracture de glène. L'objectif primaire était de définir les

8 caractéristiques primaires d'une ostéosuture d'une fracture de type Ideberg I par rapport à

9 un groupe témoin. L'objectif secondaire était de juger de l'apport d'un vissage

10 complémentaire antéro-postérieur du fragment.

11 Matériel et méthode : 15 épaules cadavériques fraîches ont été utilisées et réparties en 2

12 groupes : 5 pour le groupe témoin et 10 pour le groupe ostéosynthèse, avec la réalisation

13 séquentielle d'une ostéosuture puis d'une ostéosure+vissage. Une fracture de la partie

14 inférieure de la glène supérieure à $30 \%$ de la surface était réalisée.

15 Résultats: La charge à la rupture du groupe témoin était de $457 \mathrm{~N}$, de $277 \mathrm{~N}$ pour 16 l'ostéosuture et de $325 \mathrm{~N}$ pour l'ostéosuture+vissage. Pour ce qui est de la rigidité des 17 montages, elle était de $26,2 \mathrm{~N} / \mathrm{mm}$ pour les épaules intactes, de $14,6 \mathrm{~N} / \mathrm{mm}$ pour 18 l'ostéosuture et de $24,6 \mathrm{~N} / \mathrm{mm}$ pour l'ostéosuture+vis. La différence était significative entre 19 les 2 techniques d'ostéosynthèse, pour la charge à la rupture $(p=0,02)$ et pour la rigidité $20 \quad(p=0,001)$.

21 Discussion/conclusion: Cette étude a montré que l'ostéosuture seule permettait de 22 restaurer l'anatomie, mais en aucun la résistance mécanique de la glène native. L'ajout d'un 23 vissage améliorait significativement le montage. 
26 Niveau de preuve : IV, étude fondamentale

27

28 Mots-clés :

29 Epaule / Fracture glène / ostéosynthèse / biomécanique / model

30

31

32 


\section{Introduction}

Les luxations glénohumérales surviennent le plus souvent en position d'armé du bras, c'està-dire en abduction et en rotation latérale [1-7]. II en résulte une avulsion des structures capsulolabrales antérieures du rebord glénoïdien [6] ; ces lésions peuvent être associées à une lésion osseuse. Dans certaines conditions : mécanisme du traumatisme, âge du patient, ou niveau d'ostéoporose, l'énergie du traumatisme peut entrainer une véritable fracture articulaire de la partie antéro-inférieure de la cavité glénoïdale, fracture de type Ideberg IA [8-10]. Généralement ces fractures sont traitées orthopédiquement, mais en cas de fragment volumineux et de déplacement important (surface articulaire du fragment supérieure à $20 \%$ et déplacement supérieur à $10 \mathrm{~mm}$ [11]), l'évolution se fait vers la récidive de l'instabilité et vers une dégénérescence arthrosique $[9,11,12]$. Jusque récemment, l'ostéosynthèse chirurgicale était recommandée [13-16], mais la nécessité d'une désinsertion-suture du tendon du muscle sub-scapulaire, a favorisé le développement de l'ostéosynthèse arthroscopique de ce type de fracture par ancres de réinsertion. Cette technique donne de bons résultats cliniques et radiologiques [17-22]. Les avantages de ces techniques arthroscopiques sont $1 /$ d'améliorer la qualité de la réduction du fragment articulaire, 2/ de limiter la dissection des tissus et 3/ de diminuer la morbidité [23]. Cependant, il n'y a pas dans la littérature d'évaluation biomécanique de ces techniques arthroscopiques, nécessaires pourtant à l'évaluation des risques de déplacement secondaire et des recommandations post-opératoires.

L'objectif primaire était de définir les caractéristiques mécaniques primaires d’une ostéosuture d'une fracture de type Ideberg IA par rapport à un groupe témoin (épaules natives). L'objectif secondaire était de juger de l'apport d'un vissage complémentaire antéro-postérieur du fragment. L'hypothèse de cette étude était que l'ostéosuture 
permettait de stabiliser une fracture articulaire de la fosse glenoïdale antéro-inférieure de type Ideberg IA.

(1)

0

\section{Matériel et méthodes}

Nous avons utilisé un modèle de luxation antérieure de l'épaule déjà publié [24].

\section{Dissection}

Quinze épaules cadavériques fraiches, issues du don du corps fait à l'Institut d'Anatomie de la faculté de médecine de Strasbourg, ont été utilisées pour cette étude ( 9 provenant de sujets masculins). L'âge moyen des sujets était de 76 ans (69-82 ans). Avant toute préparation, chaque épaule a été radiographiée (avec l'humérus de face pour calculer l'index de Tingart permettant d'homogénéiser les groupes en fonction du niveau d'ostéoporose du sujet [25]) et une arthroscopie par voie postérieure a été réalisée pour éliminer toutes les épaules présentant des lésions arthrosiques ou des structures capsulo-labrales antérieures. Puis les pièces ont été tirées au sort et réparties en 2 groupes: 1) 5 pour le "groupe contrôle » et 2) 10 pour le " groupe reconstruction ».

L'ensemble des tissus a été disséqué pour ne laisser que $15 \mathrm{~cm}$ d'humérus, la capsule articulaire gléno-humérale et les tendons de la coiffe des rotateurs. Deux fils de traction ont été mis sur le tendon du muscle sub-scapulaire (Ethibond $n^{\circ} 6$, Ethicon, Somerville, NJ). Dans le "groupe reconstruction ", une fracture de la partie antéro-inférieure de la cavité glénoïdale (entre 3 et 6 h) d'une surface de près de $30 \%$ a été créée à l'ostéotome, sous contrôle radioscopique. La continuité capsulo-périostée et l'intégrité labrale ont été préservées. Dans un premier temps, l'ostéosuture a été réalisée par des points simples appuyés sur 2 à 3 ancres résorbables de diamètre 3.5mm en PLLA (Panalok, Mitek, Norwood, 
$81 \mathrm{MA}$ ) en fonction de la longueur de trait d'ostéotomie (variation en fonction de la 82 morphologie du sujet). Toutes les réparations ont été réalisées directement, en laissant le 83 tendon du muscle sub-scapulaire relevé. Après réalisation du test, la même épaule a été 84 réutilisée. Une nouvelle réparation a été réalisée, selon le même protocole, mais avec une

ancre de plus gros diamètre, insérée par le même orifice de perçage que la première ancre, à la partie crâniale et à la partie caudale de la fracture. Une vis canulée de diamètre 3,5mm a été ajoutée à l'ostéosuture, perpendiculaire au trait de fracture, et au centre de ce dernier, permettant une fixation bi-corticale complémentaire du fragment.

\section{$\underline{\text { Réalisation des tests }}$}

Les tests ont été réalisés sur une machine d'essais INSTRON (INSTRON 8500 plus, INSTRON corporation, High Wycombe, Buckinghamshire, United Kingdom). Les épaules ont été positionnées sur un montage permettant $80^{\circ}$ d'abduction dans le plan de la scapula et $90^{\circ}$ de rotation latérale de l'humérus [26], pour simuler la position d'armé du bras. La scapula était fixée entre 2 plaques et une broche était passée au travers de l'humérus pour maintenir la position (Figure 1). La précharge de $20 \mathrm{~N}$ était alors appliquée au tendon du muscle sub-scapulaire par l'intermédiaire des 2 fils pré-positionnés. Une précharge de $10 \mathrm{~N}$ était appliquée au montage par le vérin de la machine d'essais à la face postérieure de la tête humérale. Ce n'était qu'à ce moment-là que la position de l'humérus était définitivement fixée en verrouillant la broche.

La tête humérale était alors translatée antérieurement à la vitesse de $160 \mathrm{~mm} / \mathrm{s}$. La charge et le déplacement étaient enregistrés. Les 10 pièces préparées par ostéosuture étaient testées, puis les mêmes pièces cadavériques ont été re-testées avec la vis complémentaire en association de l'ostéosuture. 
104 Ont été analysés : 1 / le mode d'échec, 2 / le site de rupture, 3/ la charge à la rupture, 4/ et la 105 rigidité qui correspondait à la pente de le partie linéaire de la courbe 106 contrainte/déplacement.

107 Durant toute l'expérimentation, les pièces étaient hydratées.

108 Analyse statistique

109 Une analyse unidirectionnelle de variance a été utilisée pour comparer les 2 techniques 110 d'ostéosynthèse au groupe témoin. La comparaison des résultats des ostéosynthèses 111 successives sur la même épaule a été faite à l'aide d'un test non paramétrique de Wilcoxon. 112 Le risque alpha chois était de 5\%. Le logiciel XLstat 2007 (Addinsoft, Paris, France) a été 113 utilisé.

\section{Résultats}

117 Surface du fragment

118 Toutes les fractures concernaient plus d'un tiers de la surface articulaire inférieure (calcul

119 effectué selon la technique décrite par Sugaya et al. [12]).

\section{Type d'échec}

121 - Dans tous les cas du groupe témoin une rupture capsulo-labrale antérieure a été

122 observée (de 3 à 6 heures). Nous n'avons jamais observé de fracture du rebord antérieur

123 de la cavité glénoïdale, ni de rupture capsulaire au col de l’humérus.

124 - Dans le groupe ostéosuture simple, nous n’avons observé aucune fracture sur les points

125 d'ancrage. Dans tous les cas une rupture des fils de réinsertion avec un déplacement du 126 fragment a été constatée (figure 2). 
- Pour le groupe ostéosuture+vis, une rupture capsulaire a systématiquement été constatée, avec fragmentation du bord antérieur de la glène vissé.

\section{Caractéristiques mécaniques}

La charge à la rupture et la rigidité sont résumées dans le tableau 1.

La comparaison des résultats montre qu'il y a une diminution significative de la résistance mécanique $(p=0,001)$ et de la rigidité $(p=0,001)$ entre le groupe témoin et les 2 types d'ostéosynthèse. L'ajout d'une vis améliore significativement la charge à la rupture $(p=0,02)$ et la rigidité $(p=0,001)$ par rapport au groupe ostéosuture isolée.

\section{Discussion}

Les données obtenues pour le groupe témoin (457N) sont comparables à celles déjà publiées [24], ce qui autorise la comparaison des données avec le groupe des ostéosynthèses. Cette étude démontre clairement que la technique athroscopique d'ostéosuture des fractures de glène type Ideberg IA permet de restaurer l'anatomie de la cavité glénoïdale de la scapula, mais en aucun cas de restaurer les caractéristiques mécaniques primaires de la cavité glénoïdale native. L'ajout d'un vissage antéro-postérieur améliore significativement la stabilité mécanique du montage, tant sur la charge à la rupture $(p=0,02)$ que sur la rigidité $(p=0,001)$. II nous faut par ailleurs signaler que dans le groupe des ostéosutures, les données de charge à la rupture et de rigidité, écartypes sont beaucoup plus faibles que dans les deux autres groupes. Ceci est probablement expliqué par le fait que le montage ancres + vis étant très rigide avec la vis, c'est plus le matériel qui a été testé, que le montage en luimême. Cette étude ne permet pas de répondre à la question du fragment minimum qui permet l'adjonction d'une vis au montage d'ostéosuture. 
152 Comme seulement un tiers de la tête humérale est en contact avec la surface articulaire 153 glénoïdienne, cette articulation n'est que partiellement contrainte par ses rapports osseux. 154 Cependant les lésions de la partie inférieure de la cavité glénoïdale rendent l'articulation 155 gléno-humérale hautement instable en cas de fracture articulaire déplacée. Par ailleurs la 156 stabilité est également assurée par les structures ligamentaires antérieures [5, 27]. Ainsi il 157 semble nécessaire non seulement de réduire le fragment articulaire, mais également de fixer 158 en position anatomique les structures capsulo-labrales antérieures assurant un contrôle 159 osseux et ligamentaire articulaire. C'est probablement sur ce point que l'arthroscopie 160 permet une amélioration de la qualité et de la stabilité des réparations, car elle permet la 161 réparation des lésions associées tels que les ruptures partielles ou transfixiantes de la coiffe, 162 et/ou les extensions supérieure ou postérieure de la lésion labrale (jusqu'à 78.3\% dans la 163 série de Scheibel et al. [28]) et une meilleure fixation des structures ligamentaires.

165 Cameron [21] a été le premier à proposer une ostéosynthèse arthroscopique des fractures 166 articulaires de la cavité glénoïdale à l'aide d'une vis canulée de diamètre 3,5mm. Par la suite, 167 il a été montré qu'une ostéosuture par ancre permettait une bonne stabilisation des 168 fragments pour obtenir une consolidation en position anatomique [17-22]. Cependant, 169 toutes ces études ne concernaient que des fragments inférieurs à $20 \%$ de la surface 170 glénoïdienne. Scheibel et al. (27) ont proposé l'utilisation d'une vis résorbable comme 171 moyen de fixation du fragment osseux. Avec un taux de consolidation osseuse de $100 \%$, ces 172 auteurs suggèrent qu'en plus de la stabilité, l'adjonction d'une vis augmente la compression 173 du foyer de fracture et favorise donc sa consolidation. L'alternative à l'utilisation d'une vis 174 est la réalisation d'une stabilisation en double rang [29]. Ces auteurs ont montré par une 
175 étude cadavérique, que cette technique d'ostéosynthèse offre une stabilité supérieure à une

176 réparation par ancre insérée sur le rebord antérieur de la glène (réparation simple rang,

177 habituellement réalisée).

178

179 Limites de l'étude :

180 Comme tous les modèles cadavériques, le nombre de cas est limité au minimum

181 statistiquement validé pour l'utilisation des tests non paramétriques, et cela pour des

182 questions de disponibilité de spécimens. Par ailleurs, cette étude a été réalisée sur des

183 pièces dont l'âge des donneurs ne correspond pas forcément à l'âge des patients traités

184 pour ce type de traumatisme. Elle ne peut caractériser que la résistance mécanique primaire

185 à T0 du montage, et ne tient absolument pas compte des phénomènes de remodelage et de

186 cicatrisation. Nous n'avons pas non plus testé différents types de suture, notamment les fils

187 non résorbables de nouvelle génération. De même, le degré de déformation plastique de la

188 capsule articulaire n'est pas non plus évaluable in-vivo et ne peut donc pas être

189 expérimentalement reproduit. Enfin, le mode de luxation, par application d'une force de

190 translation postéro-antérieure, n'est pas toujours le reflet de la réalité et d'autres

191 mécanismes sont rencontrés en pratique clinique. De ce fait, cette étude ne peut pas non

192 plus évaluer les lésions associées à la fracture/luxation et leurs conséquences sur la stabilité

193 du montage.

194

195

196 Conclusions

197 L'ostéosuture arthroscopique des fractures articulaires de type Ideberg IA semble permettre

198 de stabiliser correctement le fragment antéro-inférieur. L'adjonction d'une vis améliore 
199 significativement la résistance du montage. Ces montages ne permettent cependant pas de 200 restaurer les caractéristiques mécaniques primaires de la cavité glénoïdale native. II nous 201 semble donc nécessaire d'immobiliser les patients en post-opératoire.

202 
204 PhC : consultant et développement Wright-Tornier, rédacteur associé de l'OTSR

205 FA : aucun conflit à déclarer.

206 NB : consultant Smith and Nephev, Wright-Tornier, Stryker - Royalties SBM

207 ME : consultant éducation et développement Depuy-Synthes, Lepine, Symbios, Newclip, 208 Amplitude ${ }^{\circledR}$, Rédacteur Associé des Conférences d’Enseignement de la SOFCOT 209 TB :

210 Sources de financement : aucune

211 Contribution des auteurs : les co-auteurs ont contribué à l'élaboration, la validation et la 212 rédaction du manuscrit 
214 Légende des figures

215 Figure 1 : Banc d'essais avec positionnement en armé du bras de l'articulation scapulo216 humérale. Les fils de traction sont positionnés dans le tendons du muscle sub-scapulaire $217(\mathrm{SC})$.

219 Figure 2 : Rupture des fils de réinsertion avec un déplacement du fragment (reste attaché à 220 la capsule).

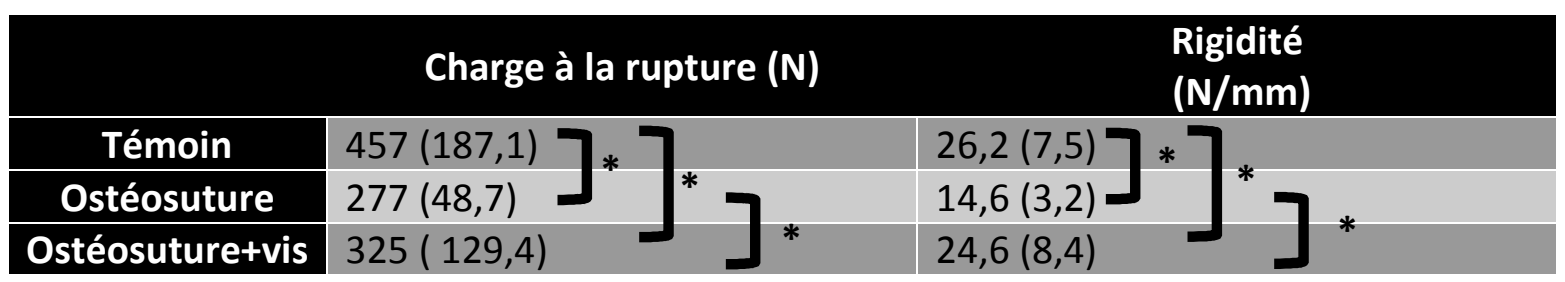

223 Tableau 1 : Résultats moyens de la charge à la rupture et de la rigidité pour le groupe témoin

224 et pour les 2 types d'ostéosynthèse. L'écart-type est entre parenthèse. * Signale des 225 différences significatives ( $p$ dans le texte). 
229 [1] Branch T, Lawton R, Lobst C, Hutton W. The role of glenohumeral capsular ligaments in 230 internal and external rotation of the humerus. Am J Sports Med. 1995;23:632-7.

231 [2] Matsen F, Fu F, Hawkins R. The shoulder : A balance of mobility and stability. In: Matsen 232 F, Fu F, Hawkins R, editors. The shoulder : A balance of mobility and stability. Rosemont, IL: 233 American Academy of Orthopaedic Surgeons; 1993. p. 279-304.

234 [3] Moseley H, Overgaard B. The anterior capsular mechanism in recurent anterior 235 dislocation of the shoulder. J Bone Joint surg [Br]. 1962;44-B:913-27.

236 [4] Ovesen J, Nielsen S. Stability of the shoulder joint : cadaver study of stabilizing structures. 237 Acta Orthop Scand. 1985;56:149-51.

238 [5] Terry G, Hammon D, France P, Norwood L. The stabilizing function of passive shoulder 239 restraints. Am J Sports Med. 1991;19:26-34.

240 [6] Bankart A. The pathology and treatment of recurrent dislocation of the shoulder joint. $\mathrm{Br}$ 241 J Surg. 1938;26:23-9.

242 [7] Perry J. Anatomy and biomechanics of the shoulder in throwing, swimming, gymnastics 243 and tennis. Clin Sports Med. 1983;2:247-70.

244 [8] Ideberg R, Grevsten S, Larsson S. Epidemiology of scapular fractures Incidence and 245 classification of 338 fractures. Acta Orthop Scand. 1995;66:395-7.

246 [9] Ideberg R. Unusual glenoid fractures: a report on 92 cases. . Acta Orthop Scand. $247 \quad 1987 ; 58: 191-2$.

248 [10] Ideberg R. Fractures of the scapula involving the glenoid fossa. In: Bateman J, Welsh R, 249 editors. Surgery of the shoulder. Philadelphia: B.C. Decker; 1984. p. 63-6.

250 [11] De Palma A. Fractures and fracture-dislocations of the shoulder girdle. Philadelphia: JB 251 Lippincott; 1983. 
252 [12] Sugaya H, Moriishi J, Dohi M, Kon Y, Tsuchiya A. Glenoid rim morphology in recurrent 253 anterior glenohumeral instability. J Bone joint Surg. 2003;85(A):878-84.

254 [13] Mayo K, Benirschke S, Mast J. Displaced fractures of the glenoid fossa. Results of open 255 reduction and internal fixation. . Clin Orthop Relat Res. 1998;347:122-30.

256 [14] Schandelmaier P, Blauth M, Schneider C, Krettek C. Fractures of the glenoid treated by 257 operation. A 5- to 23-year follow-up of 22 cases. J Bone Joint Surg. 2002;84(B):173-7.

258 [15] Sinha J, Miller A. Fixation of fractures of the glenoid rim. Injury. 1992;23:418-9.

259 [16] Kavanagh B, Bradway J, Cofield R. Open reduction and internal fixation of displaced 260 intra-articular fractures of the glenoid fossa. J Bone Joint Surg. 1993;75(Am):479-84.

261 [17] Sugaya H, Kon Y, Tsuchiya A. Arthroscopic Repair of Glenoid Fractures Using Suture 262 Anchors. Arthroscopy. 2005;21:635.e1-.e5.

263 [18] Bauer T, Abadie O, Hardy P. Arthroscopic treatment of glenoid fractures. Arthroscopy. 264 2006;22:569.e1-6.

265 [19] Tauber M, Moursy M, Eppel M, Koller H, Resch H. Arthroscopic screw fixation of large 266 anterior glenoid fractures. Knee Surg Sports Traumatol Arthrosc. 2008;16:326-32.

267 [20] Gigante A, Marinelli M, Verdenelli A, Lupetti E, Greco F. Arthroscopy-assisted reduction 268 and percutaneous fixation of a multiple glenoid fracture. Knee Surg Sports Traumatol 269 Arthrosc. 2003;11:112-5.

270 [21] Cameron S. Arthroscopic reduction and internal fixation of an anterior glenoid fracture. 271 Arthroscopy. 1998;14:743-6.

272 [22] Porcellini G, Campi F, Paladini P. Arthroscopic approach to acute bony Bankart lesion. 273 Arthroscopy. 2002;18:764-9. 
274 [23] Marsland D, Ahmed H. Arthroscopically assisted fixation of glenoid fractures: A cadaver 275 study to show potential applications of percutaneous screw insertion and anatomic risks. J 276 Shoulder Elbow Surg. 2011;20:481-90.

277 [24] clavert P, Kempf J, Kahn J. Biomechanics of open Bankart and coracoid abutment 278 procedures in a human cadaveric shoulder model. J Shoulder Elbow Surg. 2009;18:69-74.

279 [25] Tingart M, Apreleva M, von Stechow D, Zurakowski D, Warner J. The cortical thickness 280 of the proximal humeral diaphysis predicts bone mineral density of the proximal humerus. J 281 Bone Joint Surg. 2003;85(Br):611-7.

282 [26] Johnston T. The movement of the shoulder joint. Aplea for the use for 'the plan of the 283 scapula' as the reference for movement occuring at the humero-scapula joint. Br J Surg. $284 \quad 1937 ; 25: 252-60$.

285 [27] Warner J, Deng X, Warren R, Torzilli P. Static capsuloligamentous restraints to superior286 inferior translation of the glenohumeral joint. Am J Sports Med. 1992;20:675-85.

287 [28] Scheibel M, Hug K, Gerhardt C, Krueger D. Arthroscopic reduction and fixation of large 288 solitary and multifragmented anterior glenoid rim fractures. J Shoulder Elbow Surg. $289 \quad 2016 ; 25: 781-90$.

290 [29] Millett P, Braun S. The "bony Bankart bridge" procedure: a new arthroscopic technique 291 for reduction and internal fixation of a bony Bankart lesion. Arthroscopy. 2009;25:102-5. 


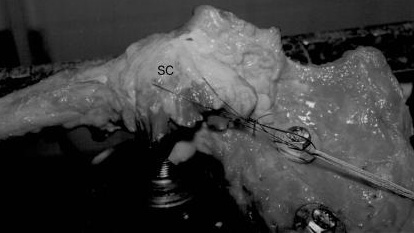



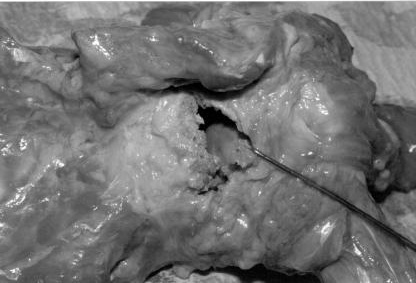